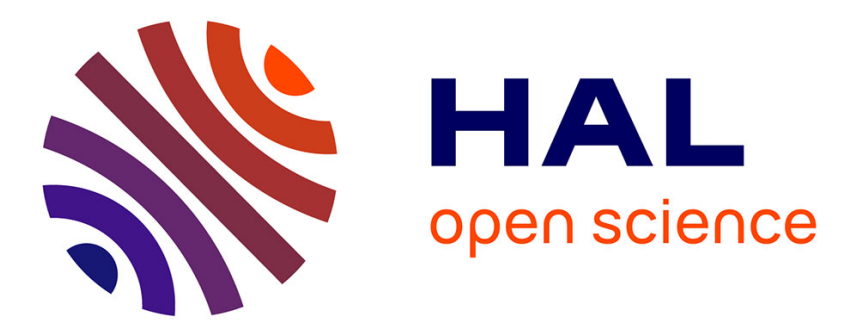

\title{
Les biotechnologies dans l'industrie agro-alimentaire: champ d'application et impact économique
}

Claude Broussolle, Gérard Brulé,. Societe Francaise d'Economie Rurale

\section{To cite this version:}

Claude Broussolle, Gérard Brulé,. Societe Francaise d'Economie Rurale. Les biotechnologies dans l'industrie agro-alimentaire: champ d'application et impact économique. Colloque SFER: Les nouvelles technologies: quels impacts sur l'agriculture et l'agro-alimentaire?, Sep 1988, Paris, France. 15 p. hal-01899854

\section{HAL Id: hal-01899854 https://hal.science/hal-01899854}

Submitted on 8 Jun 2020

HAL is a multi-disciplinary open access archive for the deposit and dissemination of scientific research documents, whether they are published or not. The documents may come from teaching and research institutions in France or abroad, or from public or private research centers.
L'archive ouverte pluridisciplinaire HAL, est destinée au dépôt et à la diffusion de documents scientifiques de niveau recherche, publiés ou non, émanant des établissements d'enseignement et de recherche français ou étrangers, des laboratoires publics ou privés.

\section{(ㅇ)(1) $\$$}

Distributed under a Creative Commons Attribution - NonCommercial - NoDerivatives| 4.0 


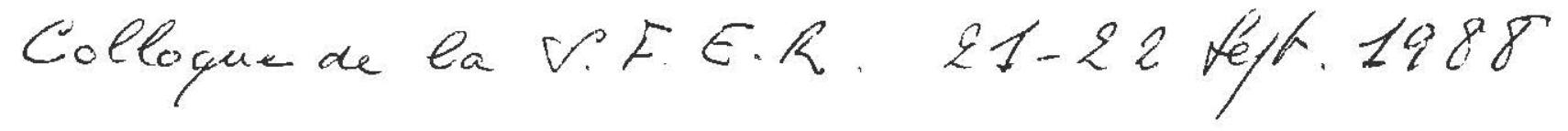

LES BIOTECENOLOGIES DANS L'INDUSTRIE AGRO-ALIYENTAIRE :

CHAMP D'APPLICATION ET RAPACT ECONOMIQUE

C. BROUSSOLLE (INRA), G. BRULE (ENSAR)

Septembre 1988

Dynamique et puissante sous de nombreux aspects, l'industrie agro-alimentaire qui a connu une croissance rapide au cours de ces trente dernières années $a$, cependant, des insuffisances, des faibiesses de structure et de rentabilité qui lui confèrent une certaine fragilité. En particulier, elle reste encore trop orientée vers la production de biens à faible valeur ajoutée pour lesquels la concurrence joue essentiellement sur les prix. En revanche, elle a des difficultés à tirer bénéfice d'une compétitivité hors coût (qualité, différenciation...) qui devralt lui permettre d'accroître ses débouchés. Il faut donc accélérer le redéploiement de l'industrie vers la fabrication de produits allmentaires plus élaborés, ce qui implique l'usage de cechnologies de plus en plus sophistiquées. Dans la compétition internationale, l'investissement n'est plus un choix mais une contrainte, et les firmes doivent assurer leur mutation à parcir de la trilogie marchésproduits-processus de transformation.

C'est dans cette perspective que doit être dérinie la stratégie à mettre en oeuvre par l'industrie agro-alimentaire si elle veut relever le défi que représentent la concurrence internationale et plus particulièrement la mise en place du marché fntérieur communautaire à l'horizon 1992. Comment préciser cette stratégie, sachant que des innovations technologiques majeures remetient en cause les structures industrielles et changent les conditions de compétitivité ? En effet, sous la poussée 
des biotechnologies, toutes les règles du jeu établies depuis des décennies sont susceptibles d'être modifiées, car leur développement. conduit à un effacement progressif des frontières entre la chimie, la pharmacie et l'agroalimentaire et à un éclatement des filières traditionnelles liant l'agriculture à l'industrie.

La difficulté à cerner tous les aspects d'une activité interdisciplinaire en mutation permanente nous a conduits, après avoir rappelé ce que sont les biotechnologies et les mutations techniques et économiques qu'elles entraînent, à utiliser comme fil directeur de l'étude, les difiérentes étapes d'un processus biotechnologique de façon à dégager les perspectives de développement qui s'offrent aux entreprises.

\section{I- LES BIOTBCHNOLOGIES : MUTATIONS TECHNIQUES ET ECONOMIQUES}

A partir d'une matière première agricole périssable et de qualité variable dans le temps et dans l'espace, l'industrie alimentaire élabore des produits stables qui doivent satisfaire à de nombreux critères de qualité. Ces critères concernent :

- les propriétés nutritionnelles (composition, valeur biologique),

- les propriétés hygiéniques (état bactériologique, absence de toxicité),

- les propriétés de services (commodité d'emploi, services à l'utilisateur, conditionnement),

- les propriétés de conservation (stabilité chimique et physique, résistance à la détérioration, protection).

Pour satisfaire à ces critères, les procédés technologiques utilisés consistent, essentiellement, à combiner et à adapter ies opérations unitaires caractéristiques de l'industrie alimentaire pour tendre vers trois objectifs principaux :

- extraire, valoriser et conserver les éléments utiles contenus dans les matières premières,

- préserver les éléments constitutifs du produit et le produit lui-même contre toute altération,

- conférer au produit toutes les caractéristiques nécessaires à son usage et aux services qu'il est appelé à rendre. 
La qualité des aliments proposés aux consommateurs s'améliore constamment grâce à une plus grande maîtrise des procédés de fabrication et au développement des technologies nouvelles. Actuellement, l'évolution scientifique et technique se caractérise par la mise en place de nouveaux outils, qu'il s'agisse de procédés de traitement et de conservation ou, plus fondamentalement, des biotechnologies. Celles-ci recouvrent les techniques utilisant les potentialités des microorganismes, des cellules végétales, ou animales, ou des fragments biochimiquement actifs qui en dérivent. Ces techniques font appel à des connaissances fondamentales, issues principalement de la biologie, la biochimie, la microbiologie, l'enzymologie et la génétique. Laur mise en oeuvre au stade industriel et commercial constitue la bio-industrie.

Le volume total du marché mondial des biotechnologies était de 15 milliards de francs en 1985. Il devrait atteindre 400 milliards en l'an 2000. La pharmacle représenterait $68 \%$ du total, 1'agro-alimentaire $20 \%, 1$ 'agriculture $11 \%, \ldots$

Dans une étude consacrée aux biotechnologies dans le monde, le CESTA distingue plusieurs modèles de développement :

- un modèle spéculatif et financier que la Suède illustre assez bien at dans lequel l'émergence des nouvelles technologies laisse espérer aux milieux financiers des bénéfices considérables. Bien que ceux-ci aient été revisés a la baisse, ce modèie perdure et constitue un puissant modèle de développement.

- un modèle industriel que $1^{\prime}$ on trouve au Japon. Les groupes industriels s'appuyant sur des activités traditionnelles diversifient leurs activités en intégrant les nouvelles technologies. Une des faiblesses du système est celle de la recherche de base. Actuellement, le Japon rattrape son retard en génie génétique.

- un "modèle de l'intégration", où recherche fondamentale, mise en oeuvre industrielle, et financement sont associés et s'articulent correctement. Les Etats-Unis illustrent avec de nombreuses faiblesses ce modele.

- un "modèle de recherche" que 1'on rencontre en Europe. Il est caractérisé par une bonne maîtrise de la recherche fondamentale et des dirficultés de transfert vers l'industrie. En France, le programme mobilisateur mis en place devrait permettre de combler notre retard dans certains domaines comme la microbiologie et le génie enzymatique.

En biotechnologie, deux types de processus sont, en effet, généralement utilisés :

- la fermentation où des organismes vivants sont multipliés dans un milieu nutritif qui secrète le produit recherché extrait ensuite du milieu de fermentation. 
- la conversion enzymatique où des enzymes (protéines douées de propriétés catalytiques) extraites de cellules animales, végétales ou microbiennes sont utilisés pour transformer un produit en un autre

Bien entendu, l'industrie agroalimentaire utilise depuis longtemps la fermentation d'un milieu naturel sous l'action de micro organismes (levures, bactéries, champignons) pour fabriquer du vin, de la bière, des fromages, ... Ce qui est nouveau, c'est que, d'une part, grâce au progrès des connaissances fondamentales dans différentes disciplines, on parvient désormais à une bonne maitrîse de ces processus et que, d'autre part, les biotechnologies permettent d'obtenir non seulement des produits consommés en l'état, mais aussi les composants de la matière première agricole; composants qui peuvent ensuite être recombinés ou utilisés comme ingrédients pour les besoins de l'industrie alimentaire.

Activité interdisciplinaire, dont la logique ne respecte pas nécessairement les frontières et les domaines sur lesquels sont construits nos systèmes scientifiques, économiques et administratifs, les biotechnologies conduisent à un effacement progressif des frontières entre la chimie, la pharmacie et l'agroalimentaire, à une remise en cause des structures industrielles et à un éclatement des filières traditionnelles liant l'agriculture à l'industrie. Par exemple, la farine de blé n'est plus regardée uniquement comme la matière première de la meunerie et de la boulangerie, mais également comme un mélange de protéines et d'amidon qu'il est facile de séparer, purifier, transformer, et incorporer dans toutes sortes d'aliments. Il en est de même du lait dont les protéines sont extraites, puis utilisées comme agents texturants, et dont le lactose peut servir de substrat dans les industrles de fermentation. Il se développe ausi progressivement une chimie des macro-molécules végétales (amidon, lignocellulose, pectines, protéines, lipides) (1).

Certains constituants biologiques présents dans ces diverses matières premières possèdent des caractéristiques physicochimiques et des propriétés qui leur sont propres, alors que d'autres ne présentent pas de spécificités particulières par rapport à leurs homologues issus d'autres matières premières. Dans le premier groupe se trouvent les protéines animales qui présentent des caractéristiques particulières qu'on ne retrouve pas dans les produits d'origine végétale; tandis que les lipides du lait et de l'oeuf ne se distinguent pas fondamentalement de celles des huiles végétales, si ce n'est par la présence en quantité plus ou moins importante de phospholipides.

(1) Les biotechnologies cortribuent à mettre à la disposition des industriels des produits agricoles mieux adaptés aux besoins des consommateurs (enrichissement des protéines du blé en lysine et du maīs en tryptophane; introduction chez l'orge de nouvelles enzymes protéolytiques pour améliorer ses qualités brassicoles, etc..). On peut considérer que $1^{\prime}$ une des retombées importantes des biotechnologies sera de contribuer à la production pour l'industrie des plantes sur mesure. L'agriculteur de demain ne fournira plus à l'usine de transformation du blé ou du maìs, mais une certaine quantité d'amidon, de protéines, de sucre... 
Les caractáristiques physicochimiques particulières de certains constituants confèrent à ces matières premières des propriétés fonctionnelles (solubilité, pouvoir moussant, pouvoir gélifiant, pouvoir émulsifiant, rétention d'eau) très recherchées de l'industrie agro-alimentaire; c'est ainsi que le lactosérum, le sérum sanguin et le blanc d'veuf sont utilisés pour leurs propriétés moussantes et gélifiantes, alors que le jaune d'oeuf l'est pour ses propriétés émulsifiantes. Ces caractéristiques leur confèrent également des propriétés nutritionnelles et physiologiques, plus ou moins bien exploitées. C'est ainsi que la valeur nutritionnelle de certains coproduits (lactosérum, sang) a longtemps été négligée et que le rôle physiologique des phosphoprotéines présentes dans 1'oeuf et dans le lait, celui des glycoprotéines que l'on trouve dans le colostrum et le blanc d'oeuf n'ont attiré 1'attention des chercheurs que depuis peu; or, ils possèdent des propriétés physiologiques, bactēriostatiques et immunologiques intéressantes.

Toutes les techniques utilisées pour exploiter ces différentes possibilités ne relèvent pas des mêmes disciplines et il est parfois dificile de tracer une frontière nette délimitant le domaine des biotechnologies. Dans les industries de l'amidon ou du sucre, par exemple, sont mêlés des processus de simple extraction, et de traitement des matières naturelles par des procédés enzymatiques. La concurrence est. ouverte entre bioconversion et synthèse chimique pour l'obtention de certains produits. Toutefois, la biochimie présente de très nets avantages pour la fabrication de produits alimentaires; qu'il s'agisse d'enzymes dont la synthèse est souvent difflcile, de la fabrication d'acides aminés pour lesquels la fermentation a l'avantage de ne produire que l'lsomère qui est en général seul assimilable par l'organisme, ou l'obtention des arômes et des parfums. Seule l'acide lactlque voit encore la coexistence des deux procédés de fabrication.

Ces perspectives de développement, aussi favorables soient-elles aux biotechnologies, ne doivent pas faire oublier qu'il existe, néanmoins, un certaln contraste entre la rapidité du progrès scientifique, d'une part et d'autre part, les délais et les limites du développement industriel. Ce décalage tient à la fois à la complexité des conditions de développement industriel, à l'utilisation des organismes modifiés, aux hésitations de la réglementation mais aussi, aux fluctuations des prix. C'est ainsi, par exemple, qu'en 1986, on a enreglstré une baisse sensible des prix européens de la lysine industrielle utilisée dans l'alimentation animale. Cette chute s'explique par la baisse des cours mondiaux du soja, stipulés en dollars. Elle a mis en difficulté les producteurs de lysine.

Les difficultés d'écoulement de certains coproduits peuvent, également, dans certains cas, freiner le développement des technologies de fractionnement de la matière première agricole. Si le problème ne se pose pas pour les coproduits protéiques il peut être soulevé pour les coproduits glucidiques et surtout pour ceux des lipides. 
Par ailleurs, on peut se demander si les consummateurs sont prêts a accepter les aliments nouveaux qu'on leur propose. En effiet, l'industrie alimentaire ne se contente pas de s'adapter à l'évolution de la demande; elle cherche, bien souvent, a l'orienter. L'enquête effectuée par le centre français de recherches en sciences sociales et économiques dans neuf pays d'Europe met en évidence des comportements qui ouvrent de larges perspectives à 1 'industrie alimentaire.

Les limites entre le naturel at $1^{\prime}$ artificiel s'estompent pour aucant que le produit "artificiel" apporte des qualités supplémentaires au produit naturel. C'est ains1 qu'en France, alors que $62 \%$ des personnes interrogées étaient, en 1976, opposées aux produits "synthétiques", il n'y en a plus que $47 \%$ en 1984 .

On assiste au développement d'une alimentation individualisée et fonctionnelle. Le besoin de variété, l'intérêt pour l'innovation culinaire sont autant d'éléments déterminants dans l'acceptation de nouveaux produits.

Le contrôle de l'alimentation par des régimes régulièrement suivis est une tendance qui se généralise. Un nombre croissant de personnes surveillent la consommation de certains produits.

L'apparition d'un courant diététique est très nette : $38 \%$ des personnes interrogées déclarent consommer des produits diététiques ou allégés et $48 \%$ manifestent un intérêt pour des produits enrichis ou améliorés.

L'alimentation des prochaines décennies cherchera avant tout à répondre à deux préoccupations majeures des consommateurs : elle devra âtre équilibrée et ne pas nuire à la santé. Il y a, en effet, une prise de conscience générale de ce qu'il convient d'appeler un juste rapport entre les apports et les besoins.

Malheureusement, les recherches en nutrition humaine ne sont pas encore suffisamment développées pour mieux définir et spécifiler les qualités des produits adaptés aux diverses demandes. C'est une lacune importante, car le développement des industries alimentaires implique que soit clairement établie une politique alimentaire. De nombreux progrès restent, d'ailleurs, à réaliser, pour améliorer la connaissance des outils biologiques (microorganismes et enzymes) dont nous disposons pour transformer la matière première agricole. Dans le domaine de l'ingénierie appliquée aux systèmes biologiques (conception de fermenteurs et de réacteurs, technologies de fermentation, procédés d'extraction et de purification des produits) de nombreux progrès restent aussi a faire. Actuellement, le développement des biotechnologies oriente et conditionne celui des biens d'équipement. On assiste d'ailleurs à un "éclatement" de ce secteur qui bénéficie des progrès réalisés dans 1'industrie chimique ("cracking alimentaire"), nucléaire (matériel d'ultra-filtration), textile (matériel de texturation et de filage), électrique (matériel de contrôle et d'automation)... L'automatisme et 
la robotique sont les éléments essentiels de cette mutation. Ainsi la mise en place de lignes de fabrication automatisées, séquencées, pour obtenir des fabrications flexibles en relation avec les différents marchés, nécessite le développement du génie des procédés ; celui-ci intègre toutes les découvertes des sciences tant biotechnologiques que physicochimiques, les automatismes et la robotique; il constitue le levier technologique nécessaire pour cette réussite de l'industriallsation du secteur agro-alimentaire de demain.

La complexité des problèmes techniques et économiques que pose le développement des biotechnologies, conduit à s'interroger sur l'aptitude des entreprises de petites et moyennes dimensions, qui constituent l'essentiel du tissu industriel français, à exploiter les voies nouvelles de la bio-industrie.

Pour essayer de répondre à cette question, nous utiliserons comme fil directeur, les différentes étapes d'un processus biotechnologique. Cette démarche nous permettra de mieux apprécier les perspectives de développement des firmes, sachant qu'il convient d'être réaliste. Si les biotechnologies offrent des perspectives de développement accru pour les industriels, elles ne sont pas sans poser quelques problèmes : d'abord les techniques à mettre en oeuvre sont complexes, d'autre part, l'identification précise des marchés n'est pas facile et la règlementation sur les produits nouveaux reste très contraignante.

\section{II- LES PERSPECTIVES DE DEVELOPPERENT AUX DIFFERENTS NIVEAUX D'UN PROCESSUS BIOTECHNOLOGIQUE}

La fabrication des produits alimentaires met en jeu deux types d'industries : une industrie de premlère transformation qui permet d'extraire de la matière première agricole une multitude de produits alimentaires intermédiaires (PAI) à forte valeur ajoutée, et une industrie de seconde transformation qui élabore à partir de ces PAI les produits alimentaires que les consommateurs attendent.

Les différentes étapes de ce processus sont représentées sur la figure 1 . 
Figure 1: LES DIFFERENTES ETAPES D'UN PROCESSUS BIOTECHNOLOGIQUE

P.A.I. *

MATIERES

PREMIERES

TECHNIQUES

SEPARATIVES

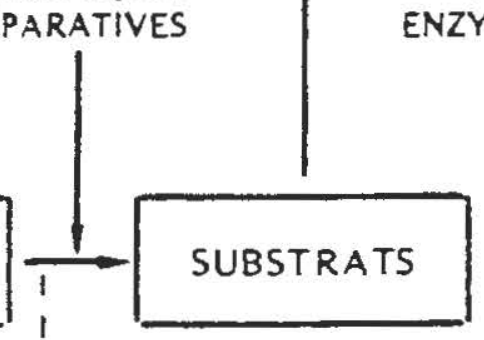

GENIE

YMATIQUE

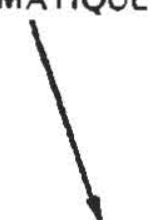

Protéines

Lait

Ovoproduit

Sang

Poisson

Légumes

Algues

1 1
Glucides

Lipides

Pectines

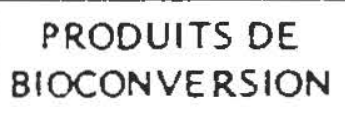

Peptides

Acides amines

Edulcorants

Acides

Arômes

Vitamines

Antibiotiques

Enzymes

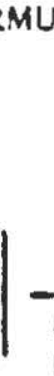

Alimentaire (charcuterie, fromagerie, confiserie, ...)

Pharmaceutique

Cosmétique

Chimique

Industrie de seconde transformation

* Produits Alimentaires Intermédiaires 


\section{Le fractionnement de la matière prentère agricole par des procédés physicochinlques}

Bien que le fractionnement proprement dit de la matlère première agricole ne fasse pas, en toute rigueur, partie des processus de la bioindustrie entendus au sens strict du terme, il n'est pas souhaitable de dissocier les biotechnologies des techniques physiques qui permettent de les mettre en oeuvre et dont elles ont souvent développé l'emplo1. En effet, les matières premières, qu'elles soient d'origine animale ou végétale, seront de moins en moins utilisées en l'état dans l'élaboration des aliments; I'examen de la composition de nombreux produits alimentaires reflète déjà cette évolution. Le fractionnement des constituants (lipides, protéines, glucides ...) des différentes matières premières offre de nombreux avantages ; il permet notamment d'offrir aux industries dites de seconde transformation (biscuiterie, salaisonnerie, confiserie, diététique...) une multitude de produits alimentaires intermédiaires (P.A.I.) dont les propriétés fonctionnelles et (ou) nutritionnelles sont améliorées par rapport à celles des matières premières initiales.

L'industrie agro-alimentaire, et notamment l'industrie laitière, ont acquis ces dernières années une très grande maîtrise des méthodes et techniques de fractionnement des constituants biologiques.

Les progrès réalisés dans le domaine du fractionnement sont à attribuer à l'amélioration des connaissances biochimlques et physicochimiques des matières premières ainsi qu'au développement des techniques nouvelles de séparation.

Parmi les techniques les plus utilisées à grande échelle et dont certaines sont toutes récentes, nous pouvons citer ia centrifugation, la filtration, la microfiltration, l'ultrafiltration, l'osmose inverse, I'évaporation, l'électrodialyse, la chromatographie. La conception modulaire de la plupart d'entre elles les rend accessibles à toute entreprise quelle que soit sa dimension.

\section{La bioconversion}

Fractionner la matière première agricole pour en extraira ses composants est une première étape qui va dans le sens d'une meilleure valorisation des ressources disponibles. Faut-il en rester là ? Evidemment non, car ce serait se priver d'une valeur ajoutée supplémentaire et laisser à d'autres la possibilité de fabriquer des produits plus élaborés. L'objectif doit être d'aller le plus loin possible dans ce processus de valorisation, tout en ayant conscience des contraintes techniques, économiques et financières qu1 limitent nécessairement les ambitions des petites at moyennes entreprises constituant l'essentiel du tissu industriel. 
Par conséquent, les nouvelles matières premières obtenues grâce à la mise en oeuvre des techniques physiques de fractionnement doivent être, dans une deuxlème phase, soumises à des traitements de nature chimique (comme, par exemple, l'hydrogénation des lipides) ou plus particulièrement, de nature blologique (fermentation, hydrolyse enzymatique), qui permettent d'améliorer leurs propriétés fonctionnelles ou nutricionnelles et, dans certains cas, de créer des molécules d'intérêt thérapeutique.

La connalssance des microorganismes et des enzymes, la maitrise de leur production et de leur utilisation à l'échelle industrielle, le transfert de compétence du génie chimique au génie biologique laissent espérer un développement rapide des techniques de bioconversion.

Les produits que la bioconversion par voie microbienne et par voie enzymatique, permet d'obtenir sont très variés :

- certains sont employés directement dans les fabrications alimentaires, comme additifs, pour les propriétés de goût, d'acidité ou de texture qu'ils y apportent.

- d'autres sont utilisés comme agents nutritifs : il s'agit notamment des acldes aminés, éléments essentiels à la croissance.

- d'autres enfin, sont employés come intermédiaires dans des processus de transformation qui fournissent des produits alimentaires.

La vole nfcroblenne permet de convertir les substrats glucidiques et des formes azotées peu élaborées en biomasse destinée à l'alimentation animale ou humaine et en biometabolites à haute valeur ajoutée. Parmi les biométabolites dignes d'intérêt, nous pouvons citer des acides organiques (acide lactique, propionique citrique...), des acides aminés (glutamique, lysine, arginine...) à usage alimentaire, des vitamines (C, B2, B 12...) et des antibiotiques à usage pharmaceutique. La mise en oeuvre simultanée de substrats glucidique, protéique et lipidique ou de leurs produits de dégradation (acides aminés, peptides, acides gras) peut conduire à l'obtention de biométabolites très variés d'intérêt aromatique (arôme de viande, de poisson, de fromage...). Les technologies utilisées en génie microbiologique sont en général de nature discontinue et mettent en oeuvre des techniques de fractionnement (centrifugation, microfiltration, ultrafiltration) bien connues du secteur agro-alimentaire; on assiste actuellement au développement de technologies à caractère continu.

Le coût de l'équipement électronique et informatique qu'implique le travail en continu d'un bioréacteur peut multiplier par 2 à 5 le coût total des installations, mais la productivité en est considérablement augmentée; elle peut être multipliée par 10 dans le cas de la fermentation lactique. 
Développer des activités de première transformation telle que la concentration des macromolécules du lait, du lactosérum, du sang, des ovoproduits, des algues, des carottes..., condult, dans tous les cas cités, à obtenir avec le concentré un coproduit composé principalement de sucres fermentescibles, de minéraux et d'azote non protéique. L'écoulement de ces coproduits peut être un frein au développement des technologies de fractionnement notamment de celles à membranes; dans la mesure où tous ces coproduits peuvent être utilisés comme support de fermentation, les constructeurs d'équipements d'ultrafiltration (Rhône-Poulenc-SFEC) ont tout intérêt à favoriser le développement du génie microbiologique.

Il corvient de remarquer que l'industrie de la fermentation est caractérisée par la lourdeur de ses investissements et l'importance des coûts énergétiques. En moyenne, le coefficient d'intensité capitalistique, rapport du coût de l'investissement au chiffre d'affaires annuel correspondant, est de l'ordre de 2 à 2,5, c'est-à-dire comparable à celui de la chimie lourde ou à celui de la sidérurgie. Quant à l'énergie, sa part dans les coûts d'exploitation est supérieure à $20 \%$.

La conversion enzyeatique, à échelle industrielle, se limite principalement à des opératlons d'hydrolyse et cela sur les différents substrats. On dispose d'enzymes permettant l'hydrolyse de lactose, de pectines de fruits, de protélnes et de lipides.

L'hydrolyse du lactose permet d'obtenir des mélanges de glucose et de galactose dont la solubilité et le pouvoir sucrant sont nettement supérieurs à ceux du substrat. Ces sucres peuvent être utilisés en l'état dans le secteur agro-alimentaire, mais dans certalnes limites, étant donné les problèmes nutritionnels posés par 1'ingestion de quantités importantes de galactose. L'originalité du lactose par rapport aux autres sources glucldiques est la présence du motif galactosique peu répandu dans la nature. L'extraction et la purification de ce sucre, qui a des applications spéclfiques en contrôle métabolique (mesure d'activité du foie), peuvent être envisagées.

L'hydrolyse des protéines pour la préparation de mélanges peptidiques à usage allmentalre, diététique, pharmaceutique, cosmétique... est beaucoup plus prometteuse. Les peptides obtenus sont utilisés pour la préparation d'aliments de réanlmation, d'aliments du premier âge, de pommades... Certaines séquences peptidiques possèdent des propriétés physiologiques particulières; nous pouvons citer par exemple les peptides phosphorylés qui jouent un rôle dans le transfert et l'absorption des minéraux et oligo-éléments; les glycopeptides qui présentent des propriétés agrégantes, d'où leur rôle bactériostatique et antiviral.

La bloconversion, qu'elle soit de nature enzymatique ou microbienne conduit à des mélanges complexes dont il faut extraire les molécules recherchées. Les industries agro-alfmentalres ont les moyens nécessaires pour effectuer ce fractionnement. 


\section{La formulation}

Ainsi que nous l'avons déjà indiqué, la grande majorité des produits alimentaires que nous consommerons demain sera élaborée non plus à partir des matières premières brutes, mais à partir d'éléments issus de plusieurs transformations, soit qu'ils proviennent du fractionnement de la matière première agricole, par des procédés physiques, soit qu'ils résultent des processus de bioconversion enzymatiques ou microbiens. Ces éléments utilisés pour leurs propriétés fonctionnelies et nutritionnelles seront "assemblés" par l'industrie et les prodults finis qu'ils permettent d'obtenir répondront aux besoins de quatre types d'alimentation :

- l'alimentation "normale"

- l'alimentation diététique

- l'alimentation spécifique

- l'alimentation thérapeutique.

Dans 1'allmentation "normale", nous mettons aussi bien les produits dits de "service" que ceux dits de festivité. Ces produits doivent apporter aux consommateurs, outre leur valeur alimentaire, un certain nombre de qualités fonctionnelles (commodité d'emploi, etc...). Au cours des prochaines années, ces aliments seront l'objet d'un certain nombre d'innovations concernant leur composition et les technologies mises en oeuvre, sans qu'il y ait de changements importants dans leur nature et leur présentation. Dans ce contexte, les nouvelles technologies seront un facteur important de compétitivité, car en assurant une composition constante et une qualité régulière aux produits, elles permettront aux entreprises de vendre sur des marchés exigeants et strictement réglementés. Elles permettront, également, de fabriquer des produits bien adaptés au goût local.

L'alimentation diététique correspond aujourd'hui à un besoin et pas seulement à une mode. Il est en effet paradoxal qu'à une époque où il existe une telle diversité alimentaire et où des progrès importants ont été réalisés dans le domaine de la nutrition humaine, on puisse parler de carences et de deséquilibres. En particulier, la consommation de fibres et de certains oligo-éléments en quantité insuffisante dans notre alimentation normale est de plus en plus souvent dénoncée par le corps médical.

Le consommateur sera de plus en plus sensible à la valeur nutritionnelle de son alimentation et à la notion de bien-être. Les produits alimentaires bien dosés en lipides, à faible teneur en glucides, à assimilation rapide, garantis en vitamines, en oligo-éléments, et à faible valeur énergétique vont connaître un développement certain, développenent accéléré par la nécessité d'engager des actions préventives dans le domaine de la santé si l'on veut équilibrer à un niveau ralsonnable les comptes de la sécurité soclale. 
Pour les produits à usage diététique, la formulation, le conditionnement, et la commercialisation peuvent être assurés par l'industrie alimentaire, d'autant plus qu'on assiste actuellement a l'insertion, dans les circuits de la grande distribution, de produits a usage diététique qui jusqu'à présent étaient exclusivement distribués dans le circuic pharmaceutique (exemples : l'aspartam, les vitamines..). Mais la formulation et le conditionnement de ces produits exigent des compétences et des règles très strictes de travail et les infrastructures industrielles existantes ne conviennent en général pas très bien car l'échelle a laquelle il faut travailler n'est pas du tout semblable. De même que les industriels laitiers quí se sont orientés dans le "cracking" alimentaire unt dû créer des structures adaptées à leurs nouvelles activités (exemples : BEL INDUSTRIE - ULN INDUSTRIE - ARMOR PROTEINES - LACTO BRETAGNE), de même, il faut envisager la mise en place de moyens de production et de commerclalisation mieux adaptés aux exigences de l'alimentation diététique.

L'alimentation "spécifique" est, en fait, une alinentation diététique qui répond aux besoins particuliers de telle ou telle catégorie de consommateurs. On y trouve les produits infantiles (laits maternisés, aliments du premier âge,...), les produits de "l'effort" (aliments du sportif), les aliments de la femme enceinte, les aliments du troisiàme åge, les aliments pour diabétiques, hypertendus,.... L'industrie alimentaire peut s'engager dans la fabrication de ce type d'aliment dans la mesure où aucun obstacle ne s'opposera à sa vente dans les circuits de la grande distribution. Bien entendu, les entreprises qui se lanceront dans ces fabrications devront bénéficier, au niveau de la formulation, du support médical, seul à même de définir les prodults et de les tester.

Pour la production des trois groupes d'aliments que nous venons de définir, il n'est pas nécessaire que les industriels de l'agro-alinentalre fassent appel à des partenaires extérieurs à leur profession s'ils recrutent un personnel compétent, et s'ils acquièrent les outils et le savoir-faire nécessalres. Néanmoins, pour réaliser ce genre d'opération, et par souci d'efficacité, les PME de l'agro-alimentaire seront, généralement, conduites à se doter d'une structure commune quí apportera l'assistance scientifique et technique nécessaire à la formulation et à la réalisation des produits.

Dans le quatrième groupe nous trouvons les alfments à usage thérapeutique. Nous y mettons les produits a usage alimentaire absorbés de façon non conventionnelle par la voie intestinale ou para-intestinale, et les produits à usage thêrapeutique proprement dits. L'industrie agro-alimentaire peut être présente dans ce secteur et doit l'être, car certalnes molécules à usage thérapeutique sont extraites de produits d'origine agricole (lysosyme, peptides à activité physiologique, lactoperoxydase, lactoferine, cytochrome,...) ; mais de telles activités ne peuvent être envisagées sans un partenaire pharmaceutique dont le rôle serait prépondérant. 


\section{Conclusion}

Sans vouloir résumer les développements qui précèdent, on se propose de rappeler en guise de conclusion, ies idées essentielles qui constituent autant d'éléments à prendre en compte dans toute réflexion concernant le développement des biotechnologies dans l'industrie agroalimentaire.

Dans un contexte caractérisé par l'évolution de pratiques alimentaires, la modernisation des processus de production et une concurrence internationale de plus en plus vive, la stratégie de l'industrie agroalimentaire est appelée à se transformer si elle veut relever le défi qui représente la mise en place d'un marché de 320 millions de consommateurs à l'horizon 1992. En effet, l'acte unique européen fixe à la CEE la réalisation d'un marché intérieur sans frontière assurant la libre circulation des personnes, des biens, des services et des capitaux. Si les européens veulent se doter des moyens de faire face à la concurrence internationale, ils seront amenés à tirer un meilleur parti de leur diversité et à jouer le jeu des avantages comparatifs (techniques et humains) de chaque pays, voire de chaque région. Dans cette perspective, les établissements industriels devront se spécialiser dans un type de produit, et sur le site où les entreprises pourront exploiter au maximum les économies d'échelle et les avantages comparatifs.

Les forces et ies faiblesses de la france par rapport aux industries de première et de deuxième transformation apparaissent nettement lorsque $l^{\prime}$ on situe les entreprises nationales aux différents niveaux d'un processus biotechnologique; processus entendu au sens large du terme, c'est-a-dire incluant les techniques physiques permettant de les mettre en oeuvre. Si l'industrie agroalimentaire maîtrise bien les techniques physiques de fractionnement, en revanche, la bioconversion par voie enzymatique n'y est pratiquée que par un nombre limité de firmes et la voie microbienne qui permet d'obtenir des biométabolites à haute valeur ajoutée est très peu utilisée. Quant à la formulation, c'est-à-dire l'assemblage d'éléments issus d'une ou plusieurs transformations, elle ne se développe que lentement.

Quelles sont les entreprises qui exploiteront demain ces différents marchés? Quelques puissantes multinationales ou une gultitude de petites et moyennes entreprises ? Naguère antinomiques, les avantages de la grande entreprise et la souplesse propre aux petites unités peuvent être conciliés et valorisés dans des ensembles structurés où se développent des relations de complémentarité, de sous-traitance,... Il n'en demeure pas moins que le risque d'une bipolarisation de 
de $1^{\prime}$ industrie agro-alimentaire est important, aussi bien au niveau de la première transformation qu'à celui de la seconde. Dans le premier cas, quelques grandes firmes affiliées aux groupes internationaux de la chimie ou de la pharmacie, disposant de capitaux et de moyens de recherche importants, maîtrisent les nouvelles technologies et contrôlent, directement, ou indirectement par le biais de licences ou de contrats de sous-traitance, une multitude de petites entreprises. Dans le second cas, quelques grandes firmes agro-alimentalres élaborent des produits bien adaptés aux besoins fonctionnels et nutritionnels des difíérentes catégories de consommateurs et laissent à d'autres le soin de fabriquer des produits banalisés où la concurrence joue essentiellement par les prix.

A cette blpolarisation de I'industrie, correspond une localisation spécifique des activités. Si le fractionnement de la matière première agricule par des procédés physiques ne peut s'envisager que sur les lieux de production, en revanche, cette contrainte est beaucoup plus légère, voire Inexistante dans certains cas, pour les processus de bioconversion. De la même façon, les éléments constitutifs des aliments de demain ne seront pas nécessalrement assemblés sur les lieux où est produite la matière première agricole, mais sur des sites où les encreprises pourront exploiter au maximum les économies d'échelle et les avantages comparatifs. En d'autre terme, la primauté de la technologie et sa fonction d'accélération du développement permettent aux grandes entreprises d'utiliser au maximum la diviston internationale du travail. 